\title{
Pseudo-thrombus mechanism in left atrial appendage visualized via transthoracic echocardiography
}

\author{
Ai Saito ${ }^{1} \cdot$ Toshimitsu Kato $^{1,2}{ }^{(0)} \cdot$ Takahiro Ikoma $^{1} \cdot$ Katsuhiko Tsunekawa $^{1} \cdot$ Noriaki Takama $^{2} \cdot$ Takao Kimura $^{1}$. \\ Masami Murakami ${ }^{1}$
}

Received: 14 May 2021 / Accepted: 26 June 2021 / Published online: 26 July 2021

(c) The Japan Society of Ultrasonics in Medicine 2021

An 81-year-old man presented with dyspnea and fever $\left(37.9^{\circ} \mathrm{C}\right)$. He had a history of paroxysmal atrial fibrillation (PAF) and cerebral infarction, treated by direct oral anticoagulant therapy. His coronavirus disease-2019 antigen test was negative. His blood test revealed elevated levels of white blood cells $(11,200)$, C-reactive protein $(10.62 \mathrm{mg} /$ $\mathrm{dL})$, D-dimer $(1.58 \mu \mathrm{g} / \mathrm{mL})$, and brain natriuretic peptide $(544.6 \mathrm{pg} / \mathrm{mL})$. Twelve-lead electrocardiography revealed normal sinus rhythm. Chest radiography revealed an increase in cardiothoracic ratio (58\%) and bilateral pulmonary consolidation and congestion. He was diagnosed with pneumonia, complicated by heart failure, and was admitted to our hospital. Transthoracic echocardiography (TTE) was performed. His left ventricular ejection fraction was preserved (60\%). On parasternal short-axis view, a low-echoic moving mass in the left atrial appendage (LAA) was observed (Fig. 1a, b). The apical two-chamber view documented the low-echoic mass $(17 \times 11 \mathrm{~mm})$ moving in the LAA, indicative of a non-artifact abnormal structure (Fig. 1c, d). A small amount of pericardial fluid was observed around the right ventricle (Fig. 1a). A thrombus was suspected, and transesophageal echocardiography (TEE) was performed. Pericardial fluid was observed around the LAA, causing it to collapse; however, no thrombus was detected. No spontaneous echo contrast (SEC) was observed in the left atrium. The pectinate muscle was visualized (Fig. 1e, f). On pulse Doppler echocardiography, the LAA blood flow was within the normal limit (filling flow: $60 \mathrm{~cm} / \mathrm{s}$ ).

Toshimitsu Kato

t-kato@gunma-u.ac.jp

1 Department of Clinical Laboratory, Gunma University Hospital, 3-39-15 Showa-machi, Maebashi, Gunma 371-8511, Japan

2 Department of Cardiovascular Medicine, Gunma University Graduate School of Medicine, 3-39-15 Showa-machi, Maebashi, Gunma 371-8511, Japan
The patient responded well to diuretics and antibiotics. He was discharged 2 weeks later.

PAF is a principal cause of cardiogenic cerebral embolism, as seen in this case [1]. Despite anticoagulation therapy, $2.7 \%$ of patients develop LAA thrombi [2]. The TTE has a poor detection rate for thrombi in the LAA. Compared to TEE, the sensitivity and specificity of TTE are low at $10 \%$ [3]. The SEC and LAA blood flow velocity $(<20 \mathrm{~cm} / \mathrm{s})$ on TEE were associated with LA thrombus $[4,5]$. However, in this case, the LAA blood flow was maintained, and SEC was not observed. Intracardiac thrombi should be differentiated from artifacts, such as side lobes, multiple reflexes, and normal structures, such as the Coumadin ridge and pectinate muscles in the LAA. The viewpoint was the slightly accumulated pericardial fluid detected on TTE (Fig. 1a). When the pericardial pressure exceeds that of the intracardiac cavity, the pectinate muscles in the LAA might be compressed by the surrounding pericardial fluid; hence, the presence of a pseudo-thrombus should be considered. 


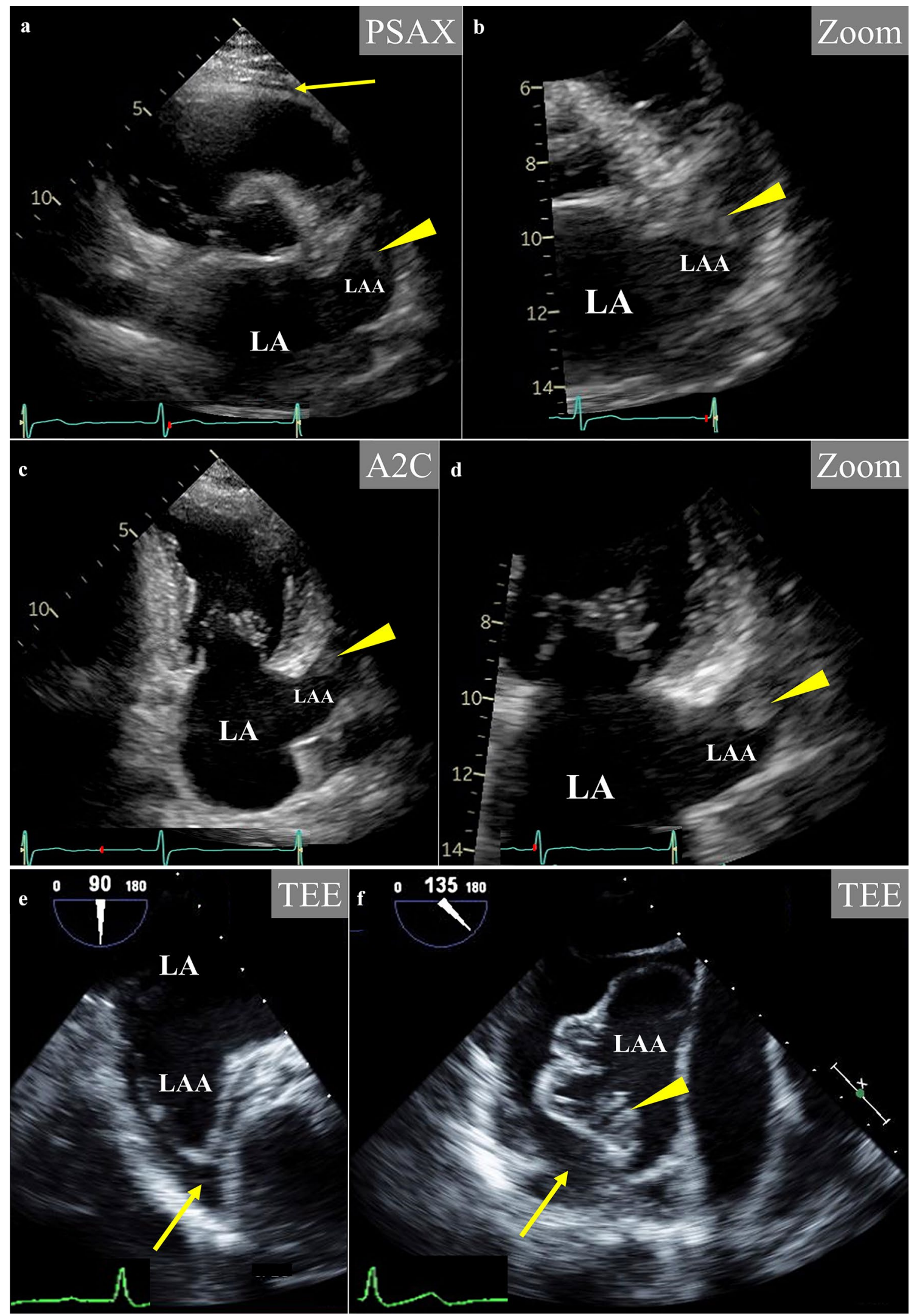


४Fig. 1 a, b Parasternal short-axis view (PSAX) and left atrial appendage (LAA) zoom view on transthoracic echocardiography (TTE) revealed a low-echoic moving mass in the LAA (yellow arrowhead). Pericardial fluid was observed around the right ventricle (yellow arrow). LA: left atrium. c, d Apical two-chamber view (A2C) and LAA zoom view on TTE also revealed a low-echoic moving mass $(17 \times 11 \mathrm{~mm})$ in the LAA, indicative of a non-artifact abnormal structure (yellow arrowhead). The LA was highly enlarged (LA volume index: $77 \mathrm{ml} / \mathrm{m}^{2}$ ). e, f Transesophageal echocardiography (TEE) revealed no thrombus in the LAA and no spontaneous echo contrast in the LA. On the other hand, pericardial fluid was observed around the LAA (yellow arrow), causing it to collapse. Moreover, the pectinate muscle in the LAA was clearly observed by the pericardial fluid (yellow arrowhead), which visually resembled a thrombus on TTE

Author contributions All authors contributed to the study's conception and design.

\section{Declarations}

Conflict of interest Ai Saito, Toshimitsu Kato, Takahiro Ikoma, Katsuhiko Tsunekawa, Noriaki Takama, Takao Kimura, and Masami Murakami declare that they have no conflicts of interest.

Ethical statements All procedures followed were in accordance with the Ethical Standards of the Responsible Committee on Human Experimentation (institutional and national) and with the Helsinki Declaration of 1964 and later versions. Informed consent was obtained from the patient.

\section{References}

1. Kaneko K, Hirono O, Fatema K, et al. Direct evidence that sustained dysfunction of left atrial appendage contributes to the occurrence of cardiogenic brain embolism in patients with paroxysmal atrial fibrillation. Intern Med. 2003;42:1077-83.

2. Kawabata M, Goya M, Sasaki T, et al. Left atrial appendage thrombi formation in Japanese non-valvular atrial fibrillation patients during anticoagulation therapy-warfarin vs direct oral anticoagulants. Circ J. 2017;81:645-51.

3. Yamamoto M, Seo Y, Kawamatsu N, et al. Complex left atrial appendage morphology and left atrial appendage thrombus formation in patients with atrial fibrillation. Circ Cardiovasc Imaging. 2014;7:337-43.

4. Fatkin D, Kelly RP, Feneley MP, et al. Relations between left atrial appendage blood flow velocity, spontaneous echocardiographic contrast and thromboembolic risk in vivo. J Am Coll Cardiol. 1994;23:961-9.

5. Donal E, Yamada H, Leclercq C, et al. The left atrial appendage, a small, blind-ended structure: a review of its echocardiographic evaluation and its clinical role. Chest. 2005;128:1853-62.

Publisher's Note Springer Nature remains neutral with regard to jurisdictional claims in published maps and institutional affiliations. 